\title{
Performance analysis of transmit antenna selection for cognitive radio systems with imperfect channel estimation
}

\author{
Peng Lan ${ }^{1 \dagger}$, Lizhen Chen ${ }^{1}$, Guowei Zhang ${ }^{2}$ and Fenggang Sun ${ }^{1 *}+$
}

\begin{abstract}
In this article, we consider a spectrum sharing cognitive radio network with a transmit antenna selection scheme, subject to the peak interference constraint of primary user (PU) and the maximum transmit power limit of secondary user (SU). Due to time-varying properties or feedback latency, the channel estimation of the link from secondary transmitter to primary receiver may be imperfect, which will result in the violation of the interference constraint at PU. Under the condition of imperfect channel state information (CSI), we derive the cumulative distribution function (CDF) and probability density function (PDF) of the SU's signal to noise ratio (SNR). Then, we obtain the closed-form expressions of average symbol error rate (SER) and capacity for the secondary system, respectively. Extensive simulations are performed with various system parameters to validate the correctness of our theoretical analysis.
\end{abstract}

Keywords: Cognitive radio, Transmit antenna selection, Imperfect channel state information, Capacity, Symbol error rate

\section{Introduction}

Cognitive radio (CR) has been considered as a promising solution to tackle the spectrum scarcity problem by allowing spectrum sharing [1]. The spectrum sharing approaches are generally classified as underlay and overlay [2]. In the spectrum underlay approach, the secondary user $(\mathrm{SU})$ coexists with primary user $(\mathrm{PU})$ in the licensed band by keeping its induced interference under a certain threshold to avoid interrupting the primary transmission. In the overlay approach, the SU detects the PU's idle spectrum for its transmission and hence no interference is induced on the PU.

Equipped with multiple antennas, SU can acquire significant performance enhancement while control the interference to PU successfully [3]. However, the benefits come at the increased hardware cost with multiple radio frequency (RF) chains. To reduce the hardware cost, the antenna selection technique has triggered significant interest for CR systems recently $[4,5]$. With negligible

\footnotetext{
*Correspondence: sunfg@sdau.edu.cn

${ }^{\dagger}$ Equal Contributors

${ }^{1}$ College of Information Science and Engineering, Shandong Agricultural

University, Daizong Road No.61, 271018 Tai'an, Shandong, China

Full list of author information is available at the end of the article
}

hardware cost, the antenna selection based CR system can obtain the most advantages of multiple antennas and the interference caused to PU can also be well-controlled.

For the traditional multi-input multi-output (MIMO) systems, various antenna selection schemes have been proposed and analyzed in terms of the average symbol error rate (SER) or capacity performance [6,7], which are also suitable to the overlay $\mathrm{CR}$ systems as $\mathrm{SU}$ does not interfere with the PU. However, such results cannot be directly applied to the underlay CR systems because the additional primary interference constraint may further affect the antenna selection criterion on the secondary transmitter. The performance analysis of the underlay CR system with transmit antenna selection (TAS) has been recently studied [8-11]. The work of [8] analyzed the SU's capacity performance with TAS under the primary interference constraint. In [9], the closed-form expression for the average capacity of the secondary link with TAS and receive maximal ratio combining (MRC) was derived under the primary interference constraint. Further in [10], the authors analyzed the performance of a MIMO cognitive system employing TAS/MRC with the peak transmit power at $\mathrm{SU}$ and the peak interference power constraint at $\mathrm{PU}$, and the closed form expressions are obtained via the 
moment generating function, in terms of the SER and the ergodic capacity, respectively. The work of [11] considered TAS scheme in spectrum sharing MIMO CR system that the antenna with the maximal ratio of the secondary channel gain to the secondary-primary interference channel gain is selected, and the thorough performance of the secondary system is analyzed in terms of outage probability, symbol error rate and ergodic capacity.

References [8-11] have all assumed that SU has full channel state information (CSI) knowledge between its transmitter and the PU receiver to protect the PU by the peak interference power constraint. However, in practice, SU may not acquire the perfect CSI of the interference link, because of several factors such as channel estimation errors and/or feedback delay, the obtained CSI may be imperfect and hence may differ from its actual value. When the imperfect CSI is used to control the interference to the PU, the interference constraint to the PU can be violated and the performance of the PU can be affected consequently. Therefore, it is important to investigate the impact of the imperfect CSI in spectrum sharing CR systems [12-15].

For underlay CR systems, the effect of imperfect CSI of the interference link between SU and PU was considered in [12], where a closed-form expression for the SU's average capacity was derived under the joint constraints of the peak interference power constraint for PU and the maximum transmit power limit for SU. The authors in [13] analyzed the cognitive underlay multihop networks with imperfect CSI and developed closed-form expressions for the secondary outage probability, bit error rate, and ergodic capacity. For cooperative cognitive MIMO networks, the authors in [14] analyzed its performance under the perfect and imperfect CSI of the secondary link condition, where the approximate expressions for outage probability and average SER is derived with primary peak interference constraint. In [15], the authors proposed to perform power control following an probabilistic interference constraint, which is derived from the point of long term statistics of the interference power.

However, to the best of our knowledge, most existing works about TAS in the underlay CR systems only focus on the performance analysis with perfect CSI of the SUPU link (e.g., See [8-11]). The imperfect CSI of the interference link is rarely investigated. Only in [16], the effect of the imperfect CSI between the secondary transmitter and the primary receiver of cognitive systems with arbitrary TAS was analyzed. However, the aforementioned work [16] focused on the achievement of the closed-expression of the capacity performance and the performance analysis for the SER performance was not involved. Besides, expect for the primary interference constraint, the SU's peak transmit power constraint is also an important parameter as all the RF power amplifiers have the maximum output power capacity [17] in the practical application. It will be shown later in our results that the neglection of the SU's maximum transmit power constraint will induce an obvious performance measurement error especially when the peak interference power constraint of the $\mathrm{PU}$ is in a large region or the interference channel from the secondary transmitter to the primary receiver is in a deep fading. In this article, a CR system employing TAS scheme at the secondary transmitter is considered. The performance of this system is analyzed for a more realistic scenario: (1) because of channel estimation errors and/or feedback delay, the channel between the secondary transmitter and the primary receiver is assumed to be imperfect, and (2) both the maximum transmit power limit at $\mathrm{SU}$ and the interference constraint at PU are taken into consideration. In this article, based on the derivation of the cumulative distribution function (CDF) and probability density function (PDF) of the SU's signal to noise ratio (SNR), the exact closed-form expressions of the average SER and capacity are obtained and validated by simulations. We can observe from the simulation results that the overestimated capacity performance caused by unlimited transmit power of SU can be corrected by our work with more reasonable performance.

To be more specific, the main contributions of this article are listed as follows.

- We characterize the effects of the imperfect CSI, the interference power constraint for SU, and the peak transmit power limit for $\mathrm{PU}$, and derive the closed-form expressions of PDF and CDF of SU's SNR with transmit antenna selection for CR networks.

- We use the back-off power control scheme to overcome the violation of interference constraint due to the imperfect CSI.

- We show that the SU's performance is greatly affected by the peak transmit power limit for PU. Therefore, the considered environment is more practical.

- We analyze the performance for secondary network in terms of the average SER and capacity and derive the closed-form expressions for CR networks, while considering all these factors, i.e., the imperfect CSI, the interference power constraint for SU, and the peak transmit power limit for PU.

The rest of this article is organized as follows. System model and problem definition is described in Section 2. Then the performance analysis in terms of the average SER and capacity different models is provided in Section 3. Simulation results are given in Section 4, and Section 5 concludes the article.

\section{System model and problem definition}

We consider an underlay spectrum sharing system where a secondary link coexists with a primary link. The 
licensed spectrum belongs to the primary link, while the secondary link can implicitly access the spectrum without violating the interference constraint of the primary link. The secondary transmitter (ST) is equipped with $N_{T}$ transmit antennas and only one RF chain. Therefore, only one antenna is selected to communicate with the one-antenna secondary destination (SD). We assume that all the links follow independent Rayleigh flat fading distribution. Let $h_{i, S D}$ and $h_{i, P}\left(i=1,2, \ldots, N_{T}\right)$ denote the channel coefficients from the $i$ th antenna of ST to SD and PD, respectively. The corresponding channel power gains $\left|h_{i, S D}\right|^{2}$ and $\left|h_{i, P}\right|^{2}$ are exponentially distributed random variables with the mean values $1 / \lambda_{D}$ and $1 / \lambda_{P}$, respectively. The thermal noise at $\mathrm{SD}$ is modeled as additive white Gaussian noise (AWGN) with zero mean and variance $\sigma^{2}$. To select a transmit antenna for SU, different selection criteria can be applied. To protect PU, a simple way is to select the antenna with the minimum interference channel gain between SU and PU, which is denoted as the interference channel gain minimum (ITEM)-based method. To enhance the secondary transmission, it is feasible to select the antenna with the maximum transmission channel gain between ST and SR [18], which is denoted as transmission channel gain maximum (TRAM)-based method. However, both the ITEM and TRAM methods only consider the interference channel or the transmission channel separately. In this article, we consider the two channels jointly and adopt the received SNR maximization as the selection criterion, since the received SNR involves the two items and determines the QoS of the secondary transmission directly.

From a practical point of view, the perfect CSI within the secondary link (i.e., $h_{i, S D}$ ) is assumed to be available at ST; however, only the partial CSI of the interference link (i.e., $\left.h_{i, P}\right)$ can be estimated. In other words, the estimated CSI of $h_{i, P}$ may differ from its actual value. The imperfect CSI of the interference link can be obtained by the SU through channel reciprocal [15] or pilot aided channel estimation [19]. In order to investigate the impact of imperfect CSI on the overall performance, we have an estimate of the channel of the form [20]

$$
\widehat{h}_{i, P}=\rho h_{i, P}+\sqrt{1-\rho^{2}} \widetilde{h}_{i, P},
$$

where $\widehat{h}_{i, P}$ is the channel estimate available at ST, and $\widetilde{h}_{i, P}$ is a complex Gaussian variable with zero mean and variance $1 / \lambda_{P}$, which is uncorrelated with $h_{i, P}$. The correlation coefficient $\rho(0 \leq \rho \leq 1)$ is a constant that determines the average quality of the channel estimate over all channel states of $h_{i, P}$.

In order to guarantee the quality of service (QoS) of the primary link, the transmit power of ST must be approximately controlled. A straightforward way is to maintain the interference caused by SU at PD below the peak interference threshold $I_{c}$. Besides, the transmit power at $\mathrm{SU}$ is also limited by the maximum transmission power $P_{m}$. The ST with the perfect CSI can suppress its power to satisfy both the constraints threshold stringently. Thus, the available power for the $i$ th transmit antenna of ST is $\min \left(P_{m}, I_{c} /\left|h_{i, p}\right|^{2}\right)$. However, when the imperfect CSI is available at the ST, as a result, the transmit power turns out to be $\min \left(P_{m}, I_{c} /\left|\widehat{h}_{i, p}\right|^{2}\right)$. Due to its uncertainty of the interference channel $\widehat{h}_{i, P}$, the obtained transmit power will possibly lead to the violation of the interference threshold at the PU. As the violation is unavoidable since the perfect CSI is not available, we use the primary interference outage probability [13] to scale the violation level, which is defined as the probability that the received interference power at the PD is higher than $I_{c}$. Accordingly, the primary interference probability Pout $_{P r i, I_{c}}$ can be denoted as

$$
\text { Pout }_{P r i, I_{c}}=\operatorname{Pr}\left(\min \left(P_{m}, \frac{I_{c}}{\left|\widehat{h}_{i, P}\right|^{2}}\right) *\left|h_{i, P}\right|^{2}>I_{c}\right),
$$

From (2), it is preferable that Pout $t_{P r i, I_{c}}$ has a small value, which means that less violation occurs. Otherwise, the primary transmission has a high probability to be disturbed by secondary link, which is not allowable in practice. To guarantee the interference probability at an acceptable level, back-off transmit power control mechanism is considered. The transmit power of the $i$ th antenna at ST can be adjusted as

$$
P_{i, b f}=\min \left(P_{m}, \varepsilon_{i} I_{c} /\left|\widehat{h}_{i, p}\right|^{2}\right),
$$

where $\varepsilon_{i}$ denotes back-off power control coefficient for the $i$ th antenna. The back-off power control coefficient $\varepsilon_{i}$ needs to satisfy the predetermined interference outage probability $P_{I}$ which is derived as [21]

$$
\begin{aligned}
P_{I}= & e^{-\beta}-e^{-\beta} Q_{1}\left(\sqrt{\frac{2 \rho^{2} \beta}{1-\rho^{2}}}, \sqrt{\frac{2 \varepsilon_{i} \beta}{1-\rho^{2}}}\right)-\frac{t}{r} Q_{1}\left(\sqrt{\frac{\beta(s-r)}{2}}, \sqrt{\frac{\beta(s+r)}{2}}\right) \\
& +\frac{1}{2}\left(1+\frac{t}{r}\right) e^{-\frac{s \beta}{2}} I_{0}\left(\frac{2 \rho \beta \sqrt{\varepsilon_{i}}}{1-\rho^{2}}\right) \\
s= & \frac{2\left(1+\varepsilon_{i}\right)}{1-\rho^{2}}, t=\frac{2\left(1-\varepsilon_{i}\right)}{1-\rho^{2}}, r=\frac{2 \sqrt{\left(1+\varepsilon_{i}\right)^{2}-4 \rho^{2} \varepsilon_{i}}}{1-\rho^{2}},
\end{aligned}
$$

where $\beta=I_{c} / P_{m}$, and $I_{0}(\cdot)$ is the zeroth-order modified Bessel function of the first kind and $Q_{1}(a, b)=$ $\int_{b}^{\infty} x e^{-\frac{x^{2}+a^{2}}{2}} I_{0}(a x) d x$ is the first-order Marcum Qfunction [22]. However, the coefficient $\varepsilon_{i}$ can not be derived as a closed form to satisfy (4), hence, the coefficient $\varepsilon_{i}$ can be numerically derived [21], with which ST can adjust its transmit power to satisfy the primary interference outage constraint. It is notable that the back-off power control coefficient $\varepsilon_{i}$ for each antenna is equal, i.e., $\varepsilon_{1}=\varepsilon_{2}=\cdots=\varepsilon_{N_{T}}=\varepsilon$. 
Through back off power control, the transmit power for all the $N_{T}$ antennas can be obtained. Then according to the received SNR maximization criterion, let $S$ denote the best transmit antenna selected from the transmit antennas set $\mathbb{T}$, and the received SNR at SD can be given as

$$
\gamma_{S}=\max _{i \in \mathbb{T}}\left(\gamma_{i}\right)=\max _{i \in \mathbb{T}}\left[\frac{\min \left(P_{m}, \varepsilon I_{c} /\left|\widehat{h}_{i, P}\right|^{2}\right)\left|h_{i, S D}\right|^{2}}{\sigma^{2}}\right]
$$

As $\left|h_{i, S D}\right|^{2}$ and $\left|\widehat{h}_{i, P}\right|^{2}$ follow exponential distribution with parameters $1 / \lambda_{D}$ and $1 / \lambda_{P}$, respectively. Therefore, the CDF $F_{\gamma_{S}}(x)$ can be written as

$$
\begin{aligned}
F_{\gamma_{S}}(x) & =P\left[\max _{i \in \mathbb{T}}\left(\frac{\min \left(P_{m}, \varepsilon I_{c} /\left|\widehat{h}_{i, P}\right|^{2}\right)\left|h_{i, S D}\right|^{2}}{\sigma^{2}}\right) \leq x\right] \\
& =\left[1-P\left(\frac{P_{m}\left|h_{i, S D}\right|^{2}}{\sigma^{2}}>x, \frac{\varepsilon I_{c}\left|h_{i, S D}\right|^{2}}{\sigma^{2}\left|\widehat{h}_{i, P}\right|^{2}}>x\right)\right]^{N_{T}} .
\end{aligned}
$$

Assume $\left|h_{i, S D}\right|^{2}=Y$ and $\left|\widehat{h}_{i, P}\right|^{2}=Z$, (7) can be further written as

$$
\begin{aligned}
F_{\gamma_{S}}(x) & =\left[1-P\left(\frac{\sigma^{2} x}{P_{m}}<Y, Z<\frac{\varepsilon I_{c} Y}{\sigma^{2} x}\right)\right]^{N_{T}} \\
& =\left[1-\int_{\frac{\sigma^{2} x}{P_{m}}}^{+\infty} \lambda_{D} \exp \left(-\lambda_{D} y\right) \int_{0}^{\frac{\varepsilon I_{c} y}{\sigma^{2} x}} \lambda_{P} \exp \left(-\lambda_{P} z\right) d z d y\right]^{N_{T}} \\
& =\left[1-\left(1-\frac{x}{x+\Omega_{2}} \exp \left(-\frac{\Omega_{2}}{\Omega_{1}}\right)\right) \exp \left(-\frac{x}{\Omega_{1}}\right)\right]^{N_{T}}
\end{aligned}
$$

where $\Omega_{1}=\frac{P_{m}}{\lambda_{D} \sigma^{2}}$ and $\Omega_{2}=\frac{\varepsilon \lambda_{P} I_{c}}{\lambda_{D} \sigma^{2}}$. By using the binomial expansion, (8) can be expressed as

$$
\begin{aligned}
F_{\gamma_{S}}(x)= & \sum_{i=0}^{N_{T}} \sum_{j=0}^{i} \sum_{k=0}^{j} C_{N_{T}}^{i} C_{i}^{j} C_{j}^{k}(-1)^{i+j+k} \\
& \times \exp \left(-j \frac{\Omega_{2}}{\Omega_{1}}\right) \Omega_{2}^{k} \exp \left(-i \frac{x}{\Omega_{1}}\right) \frac{1}{\left(x+\Omega_{2}\right)^{k}} .
\end{aligned}
$$

Taking the derivative of $F_{\gamma_{S}}(x)$ with respect to $x$, the PDF of $\gamma_{S}$ is obtained as

$$
\begin{aligned}
f_{\gamma_{S}}(x)= & \sum_{i=0}^{N_{T}} \sum_{j=0}^{i} \sum_{k=0}^{j} C_{N_{T}}^{i} C_{i}^{j} C_{j}^{k}(-1)^{i+j+k} \exp \left(-j \frac{\Omega_{2}}{\Omega_{1}}\right) \\
& \times \Omega_{2}^{k} \exp \left(-i \frac{x}{\Omega_{1}}\right) \frac{1}{\left(x+\Omega_{2}\right)^{k}}\left[\frac{i}{\Omega_{1}}+\frac{k}{x+\Omega_{2}}\right] .
\end{aligned}
$$

\section{Performance analysis}

In this section, we investigate the performance of the TAS-based underlay CR system in terms of the average SER and capacity with imperfect CSI of the link from ST to PD.

\subsection{Average symbol error rate}

The average SER is one of the most commonly used performance criterion for digital communication systems.
The straightforward expression of the average SER is given by

$$
P_{e}=\int_{0}^{+\infty} P_{S}(x) f_{\gamma_{S}}(x) d x
$$

where $P_{S}(x)$ is the instantaneous error probability in AWGN channel with SNR $x$ for the particular modulation used. Meanwhile, the SER can also be expressed as [23]

$$
P_{e}=\int_{0}^{+\infty} \frac{d P_{S}(x)}{d x} F_{\gamma_{S}}(x) d x
$$

where $\frac{d P_{S}(x)}{d x}$ denotes the derivative of the instantaneous error probability $P_{S}(x)$.

For different modulation schemes employed in practical systems, the instantaneous error probability $P_{S}(x)$ is of different forms that we summarized in Table 1 [24].

For the case $P_{S}(x)=\alpha \exp (-\beta x)$, we use the expression of the average SER in (11). By substituting (10) into (11), the average SER can be calculated as:

$$
\begin{aligned}
P_{e}= & \sum_{i=0}^{N_{T}} \sum_{j=0}^{i} \sum_{k=0}^{j} C_{N_{T}}^{i} C_{i}^{j} C_{j}^{k}(-1)^{i+j+k+1} \exp \left(-j \frac{\Omega_{2}}{\Omega_{1}}\right) \\
& \times \Omega_{2}^{k} \int_{0}^{+\infty} \alpha \exp (-\beta x) \frac{\exp \left(-i x / \Omega_{1}\right)}{\left(x+\Omega_{2}\right)^{k}}\left[\frac{i}{\Omega_{1}}+\frac{k}{x+\Omega_{2}}\right] d x .
\end{aligned}
$$

Carrying out the integral in (13), the closed form expression of the average SER for SU is obtained as:

$$
\begin{aligned}
P_{e} & =\sum_{i=1}^{N_{T}} \sum_{j=0}^{i} C_{N_{T}}^{i} C_{i}^{j}(-1)^{i+j+1} \exp \left(-j \frac{\Omega_{2}}{\Omega_{1}}\right) \frac{\alpha}{(\beta / i) \Omega_{1}+1} \\
& +\sum_{i=1}^{N_{T}} \sum_{j=1}^{i} \sum_{k=1}^{j} C_{N_{T}}^{i} C_{i}^{j} C_{j}^{k}(-1)^{i+j+k+1} \exp \left(-j \frac{\Omega_{2}}{\Omega_{1}}\right) \Omega_{2}^{k} \alpha \\
& \times\left\{\frac{i}{\Omega_{1}}\left(\beta+\frac{i}{\Omega_{1}}\right)^{k-1} \exp \left[\left(\beta+\frac{i}{\Omega_{1}}\right) \Omega_{2}\right] E_{k}\left[\left(\beta+\frac{i}{\Omega_{1}}\right) \Omega_{2}\right]\right. \\
& \left.+k\left(\beta+\frac{i}{\Omega_{1}}\right)^{k} \exp \left[\left(\beta+\frac{i}{\Omega_{1}}\right) \Omega_{2}\right] E_{k+1}\left[\left(\beta+\frac{i}{\Omega_{1}}\right) \Omega_{2}\right]\right\}
\end{aligned}
$$

Table 1 Instantaneous error probability for different modulation schemes

\begin{tabular}{lll}
\hline$P_{S}(x)$ & Modulation scheme & Parameter selection \\
\hline$\alpha \exp (-\beta x)$ & DPSK & $\alpha=0.5, \beta=1$ \\
& NCBFSK & $\alpha=0.5, \beta=0.5$ \\
$\alpha Q(\sqrt{\beta x})$ & BPSK & $\alpha=0.5, \beta=1$ \\
& BFSK & $\alpha=0.5, \beta=0.5$ \\
& MPAM & $\alpha=\frac{M-1}{M}, \beta=\frac{3 \log _{2} M}{M^{2}-1}$ \\
& MQAM & $\alpha \approx 2\left(1-\frac{1}{\sqrt{M}}\right), \beta \approx \frac{1.5}{M-1}$ \\
& MPSK & $\alpha \approx 1, \beta \approx \sin ^{2}\left(\frac{\pi}{M}\right)$ \\
\hline
\end{tabular}

Note: $Q(x)=\frac{2}{\sqrt{\pi}} \int_{x}^{+\infty} \exp \left(-t^{2}\right) d t$ 
where $E_{n}(m)$ is the exponential integral [25], defined by $E_{n}(m)=\int_{m}^{+\infty} z^{-n} \exp (-z) d z$ for $m>0$.

For the case $P_{S}(x)=\alpha Q(\sqrt{\beta x})$, we use expression (12) to obtain the average SER, i.e.,

$$
P_{e}=\alpha \sqrt{\frac{\beta}{\pi}} \int_{0}^{+\infty} \frac{\exp (-\beta x)}{\sqrt{x}} F_{\gamma_{S}}(x) d x .
$$

Then, by substituting (9) into (15), the average SER can be evaluated as

$$
\begin{aligned}
P_{e}= & \sum_{i=0}^{N_{T}} \sum_{j=0}^{i} \sum_{k=0}^{j} C_{N_{T}}^{i} C_{i}^{j} C_{j}^{k}(-1)^{i+j+k} \exp \left(-j \frac{\Omega_{2}}{\Omega_{1}}\right) \\
& \times \alpha \sqrt{\frac{\beta}{\pi}} \int_{0}^{+\infty} \frac{\exp (-\beta x)}{\sqrt{x}} \frac{\exp \left(-i x / \Omega_{1}\right)}{\left(x / \Omega_{2}+1\right)^{k}} d x \\
= & \sum_{i=0}^{N_{T}} \sum_{j=0}^{i} \sum_{k=0}^{j} C_{N_{T}}^{i} C_{i}^{j} C_{j}^{k}(-1)^{i+j+k} \exp \left(-j \frac{\Omega_{2}}{\Omega_{1}}\right) \\
& \times \alpha \sqrt{\beta \Omega_{2}} \phi\left[\frac{1}{2} ; \frac{3}{2}-k ; \sqrt{\Omega_{2}}\left(\beta+\frac{i}{\Omega_{1}}\right)\right]
\end{aligned}
$$

where $\phi(x ; y ; z)$ is the confluent hypergeometric function of the second kind, defined by the integral (9.211.4), [25]

$$
\phi(x ; y ; z)=\frac{1}{\Gamma(x)} \int_{0}^{+\infty} \exp (-z t) t^{x-1}(1+t)^{y-x-1} d t .
$$

\subsection{Average capacity}

The average capacity is also an important performance metric as it provides insight on the maximum achievable transmission rate under which the errors are recoverable. For the TAS CR system, the received SNR at SD follows a distribution with PDF given by (10). According to the definition of the instantaneous capacity $C_{\gamma_{S}}=\log _{2}\left(1+\gamma_{S}\right)$, the average capacity of SU can be expressed as

$$
R_{S}=\int_{0}^{+\infty} \log _{2}(1+x) f_{\gamma_{S}}(x) d x
$$

where $x$ denotes the instantaneous received SNR at SD. Substituting (10) into (18) and after some necessary but tedious manipulations, we can get a closed form expressions of the average capacity $R_{S}$ as

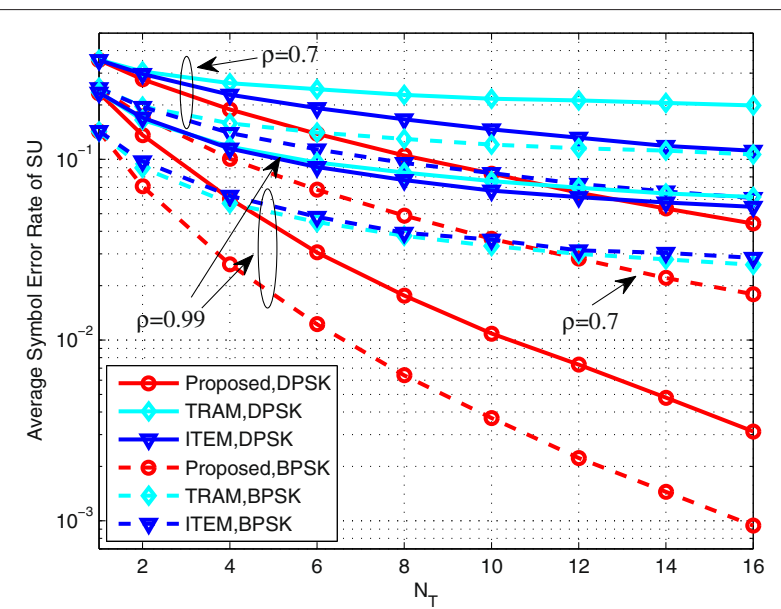

Fig. 1 The average symbol error rate vs. number of transmit antennas of SU: $N_{T}$, when $P_{m}=0 \mathrm{dBW}, I_{c}=-10 \mathrm{dBW}$, and $P_{l}=0.1$

\section{Numerical and simulation results}

In this section, we firstly compare the proposed method with TRAM and ITEM via Monte Carlo simulations to verify its effectiveness. Then, we confirm the analytical results derived in Section 3 in terms of the average SER and capacity performance, respectively, for the TASbased CR system with imperfect CSI. We consider the BPSK and DPSK modulation schemes for the performance evaluation. Throughout this section, the following system parameters are used: $\lambda_{P}=\lambda_{D}=1$ and $\sigma^{2}=-10 \mathrm{dBW}$. From Figs. 1 and 2, the proposed method provides superior performance in terms of average SER and capacity, as compared to TRAM and ITEM. As shown in Figs. 3, 4, $5,6,7,8,9$, and 10 , the analytical results and the simulation results always match exactly, which indicates that our theoretical analysis is valid and accurate.

Figures 1 and 2 depict the average SER and ergodic capacity of SU for different methods vs. the number of SU's transmit antennas $N_{T}$ when $P_{m}=0 \mathrm{dBW}, I_{c}=$ $-10 \mathrm{dBW}$, and $P_{I}=0.1$. As is shown, with the increase of transmit antenna number, the performance of both the average SER and ergodic capacity is enhanced. As

$$
\begin{aligned}
& R_{S}= \\
& \left\{\begin{array}{l}
\frac{1}{\ln 2} \sum_{i=1}^{N_{T}} \sum_{j=0}^{i} \sum_{k=0}^{j} C_{N_{T}}^{i} C_{i}^{j} C_{j}^{k}(-1)^{i+j+k+1} \exp \left(\frac{i-j}{\Omega_{1}}\right)\left(\frac{i}{\Omega_{1}}\right)^{k} E_{k+1}\left(\frac{i}{\Omega_{1}}\right), \\
\frac{1}{\ln 2} \sum_{i=1}^{N_{T}} \sum_{j=0}^{i} \sum_{k=0}^{j} C_{N_{T}}^{i} C_{i}^{j} C_{j}^{k}(-1)^{i+j+k} \exp \left(-j \frac{\Omega_{2}}{\Omega_{1}}\right)\left(\frac{\Omega_{2}}{\Omega_{2}-1}\right)^{k} \exp \left(\frac{i}{\Omega_{1}}\right) E_{i}\left(\frac{-i}{\Omega_{1}}\right) \\
+\frac{1}{\ln 2} \sum_{i=1}^{N_{T}} \sum_{j=1}^{i} \sum_{k=0}^{j} \sum_{t=1}^{k} C_{N_{T}}^{i} C_{i}^{j} C_{j}^{k}(-1)^{i+j+k} \exp \left(-j \frac{\Omega_{2}}{\Omega_{1}}\right) \Omega_{2}^{k}\left(\frac{1}{\Omega_{2}-1}\right)^{k+1-t}\left(\frac{i}{\Omega_{1}}\right)^{t-1} \\
\times \exp \left(i \frac{\Omega_{2}}{\Omega_{1}}\right) E_{t}\left(i \frac{\Omega_{2}}{\Omega_{1}}\right),
\end{array}\right.
\end{aligned}
$$




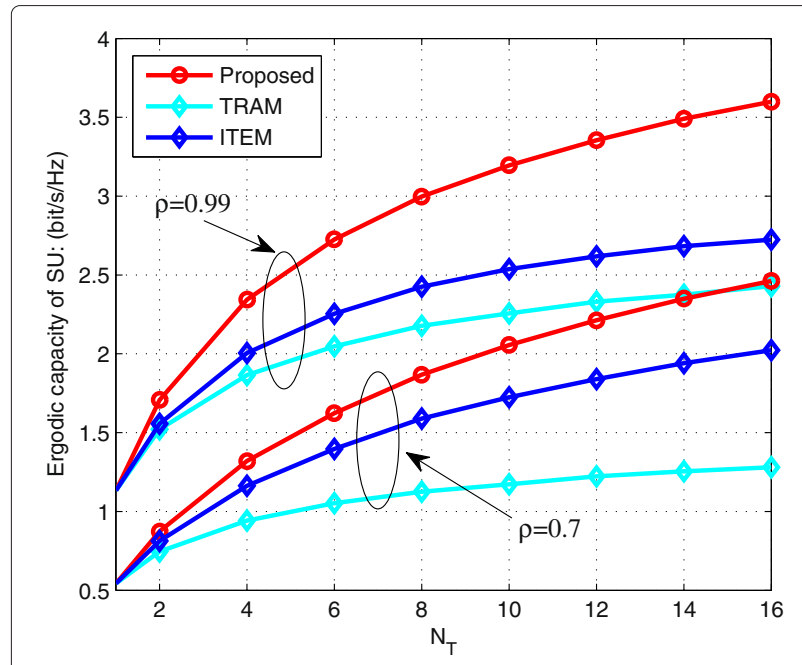

Fig. 2 The ergodic capacity vs. number of transmit antennas of SU: $N_{T}$, when $P_{m}=0 \mathrm{dBW}, I_{c}=-10 \mathrm{dBW}$, and $P_{l}=0.1$

compared to TRAM and ITEM, the proposed method provides better performance. This is because the proposed method jointly considers the interference channel between SU and PU and the transmission channel for SU. Therefore, the proposed method is superior to the other two methods.

Figures 3 and 4 show the average SER and ergodic capacity of SU with respect to different channel coefficient $\rho$ for $N_{T}=1,4$ and $I_{c}=-10,-8 \mathrm{dBW}$. As can be clearly seen, the average SER and ergodic capacity performance of SU will be improved with the increase of channel coefficient $\rho$, i.e., channel estimation quality increases. Note that the SU's performance is more sensitive to the value of $\rho$ that is close to 1 , since we observe a higher dynamic range for such values. Figures 3 and 4 also show that the

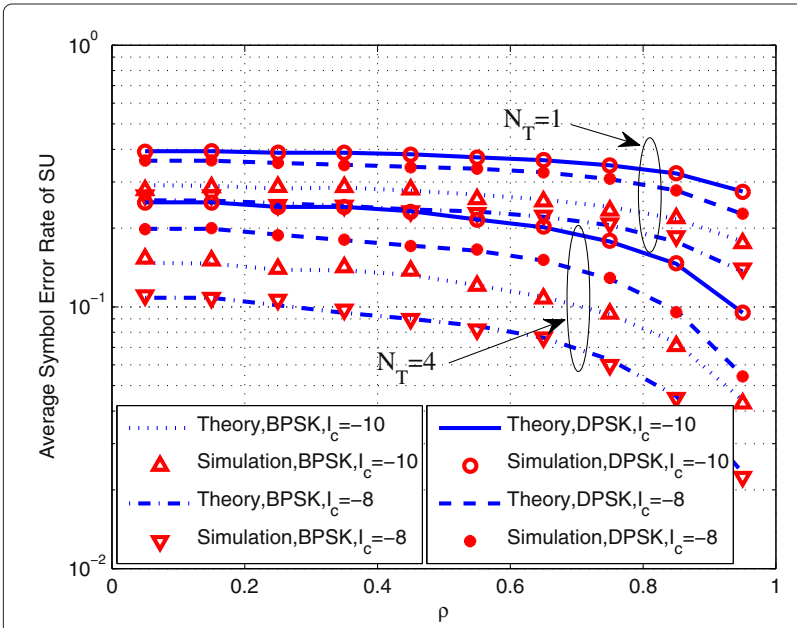

Fig. 3 The average symbol error Rate vs. channel correlation coefficient $\rho$, when $P_{m}=0 \mathrm{dBW}$ and $P_{l}=0.1$

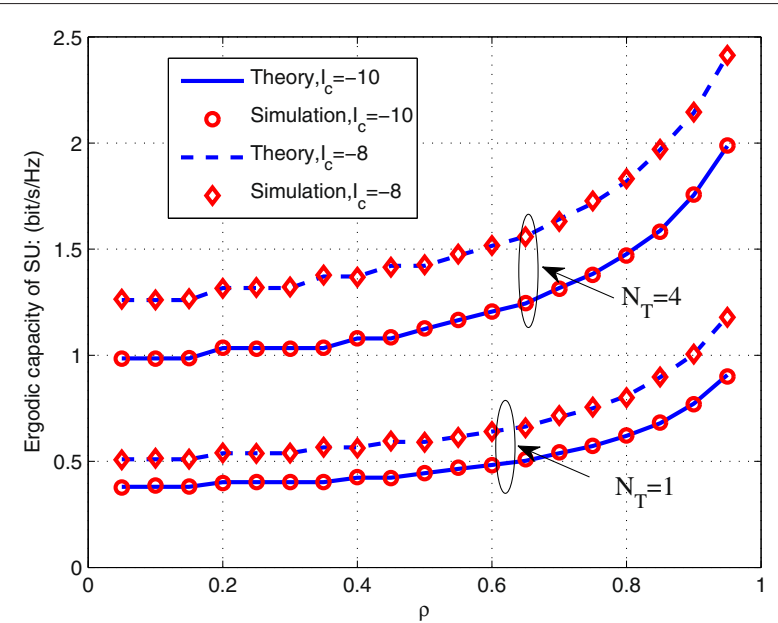

Fig. 4 The ergodic capacity vs. channel correlation coefficient $\rho$, when $P_{m}=0 \mathrm{dBW}$ and $P_{l}=0.1$

increase of $N_{T}$ and $I_{c}$ will improve the average SER and ergodic capacity of SU. This is becasue higher transmit diversity can be achieved with the increase of $N_{T}$ and more transmit power is enabled with larger $I_{c}$ according to (3).

Figures 5 and 6 show the average SER and ergodic capacity of SU with different levels of allowed interference power $I_{c}$ at PU for $N_{T}=1$, 4. In particular, to investigate the impact of the maximum transmit power limit $P_{m}$ at $\mathrm{SU}$, the cases of $P_{m}=0 \mathrm{dBW}$ and $P_{m} \rightarrow \infty$ are compared. As is shown, the performance difference between the cases with and without the maximum transmit power limit is negligible when $I_{c}$ is in the low region. With the increase of $I_{c}$, the performance of the cases without the maximum transmit power limit improves continuously. However, with the

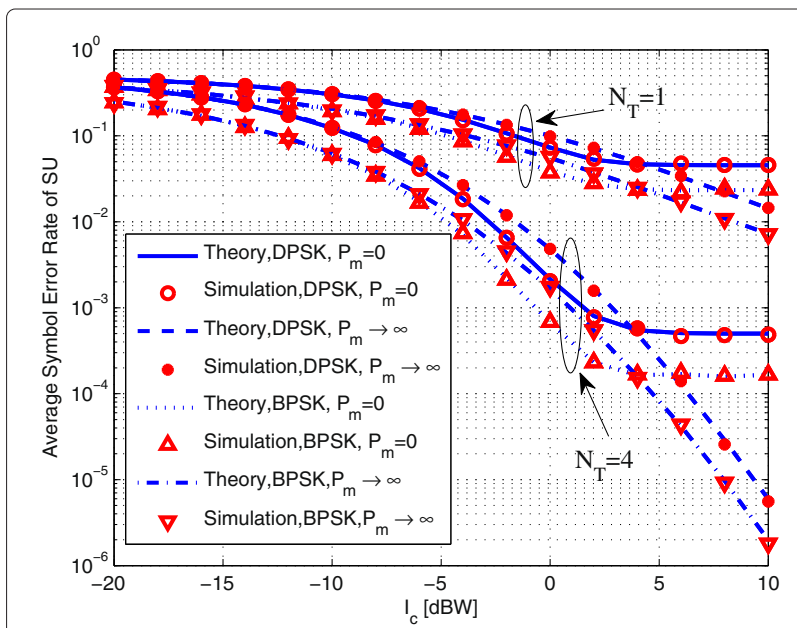

Fig. 5 The average symbol error rate vs. interference constraint: $I_{c}$ (dBW), when $\rho=0,9$ and $P_{l}=0.1$ 


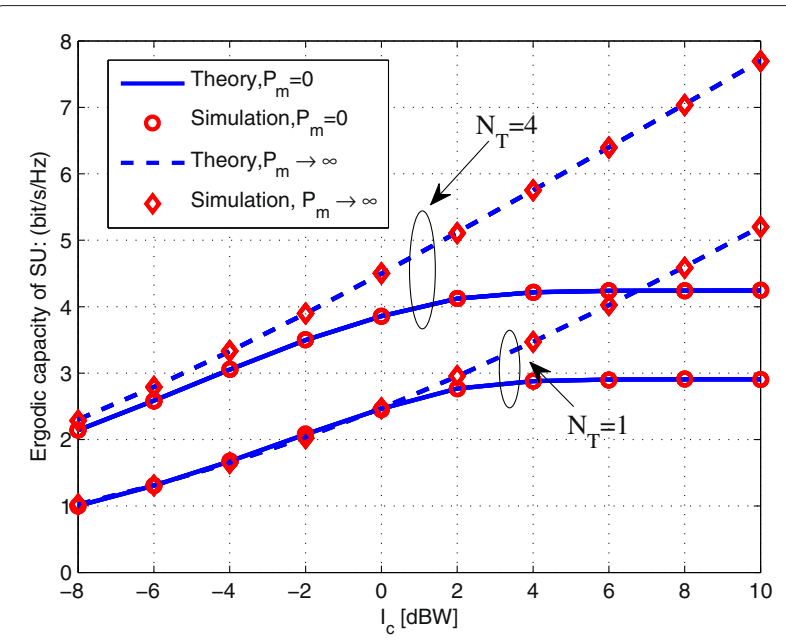

Fig. 6 The ergodic capacity vs. interference constraint: $I_{c}(d B W)$, when $\rho=0,9$ and $P_{l}=0.1$

maximum transmit power limit, the average SER and capacity of SU will firstly improve and finally achieve a performance ceiling. This phenomenon can be explained as follows: when $I_{c}$ is in the low region, the transmit power of $\mathrm{SU}$ is dominantly decided by the primary interference constraint, and when $I_{c}$ is in the high regions, the transmit power of $\mathrm{SU}$ is dominated by the maximum transmit power limit of SU. Obviously, neglecting the maximum transmit power limit will result in inaccuracy for the performance analysis of $\mathrm{SU}$ when $I_{c}$ is in high region.

In Figs. 7 and 8, we plot the average SER and ergodic capacity performance vs. the SU maximum transmit power limit $P_{m}$ for $N_{T}=1,4$ and $\rho=0.9,0.99$. As can be seen, the system performance improves with the increase

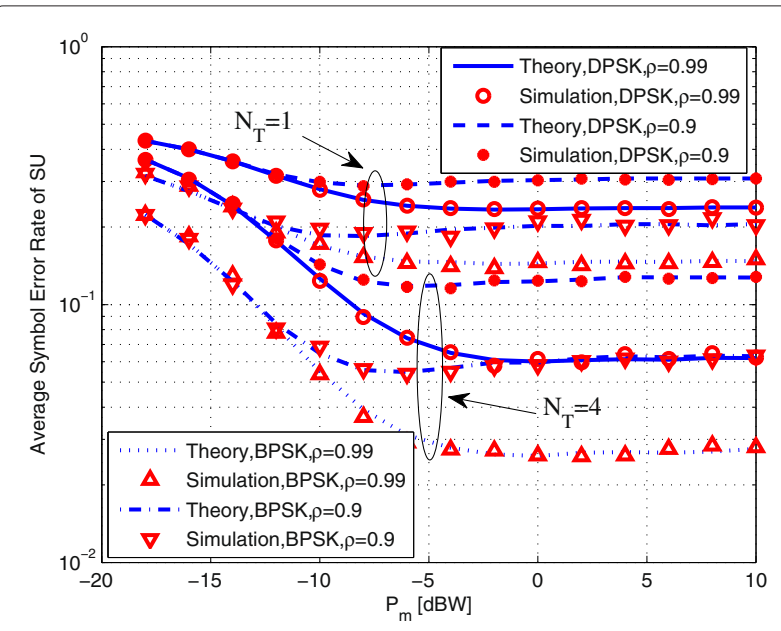

Fig. 7 The average symbol error rate vs. SU maximum transmit power limit: $P_{m}(\mathrm{dBW})$, when $I_{c}=-10 \mathrm{dBw}$ and $P_{l}=0.1$

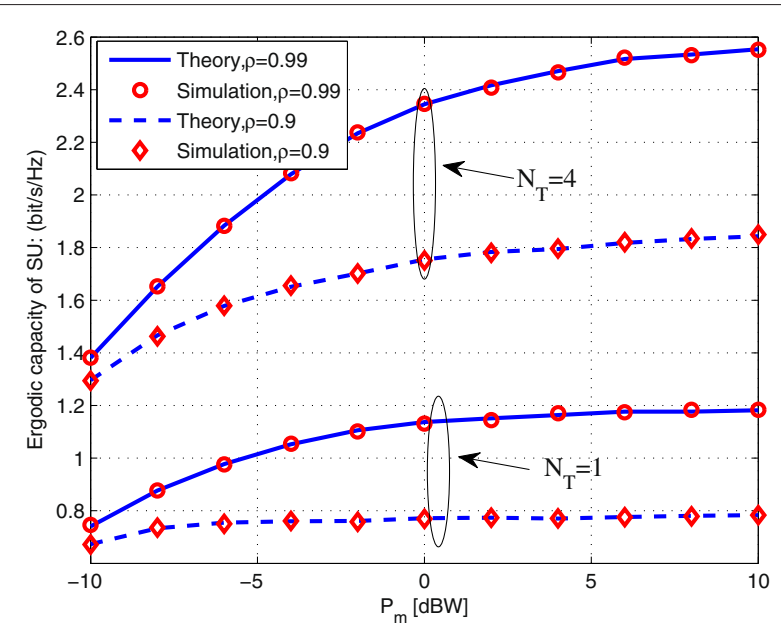

Fig. 8 The ergodic capacity vs. SU maximum transmit power limit: $P_{m}$ $(\mathrm{dBW})$, when $I_{\mathrm{c}}=-10 \mathrm{dBW}$ and $P_{l}=0.1$

of $P_{m}$. The performance ceiling achieves when $P_{m}$ is in the high regions, which is due to the SU's interference level constraint $I_{c}$. Moreover, the system performance improves with the increase of antenna number $N_{T}$ and channel coefficient $\rho$. The reasons are as follows: more transmit antennas can provide higher diversity gain and the transmit power increases as channel estimation quality $\rho$ increases.

Figures 9 and 10 show that the average SER and ergodic capacity performance versus the interference outage probability $P_{I}$ of SU for $N_{T}=1,4$ and $I_{c}=$ $-10,-8 \mathrm{dBW}$. As can be seen, with the increase of $P_{I}$, the average SER and ergodic capacity performance improves for the reason that more transmit power is allowed for the secondary transmission.

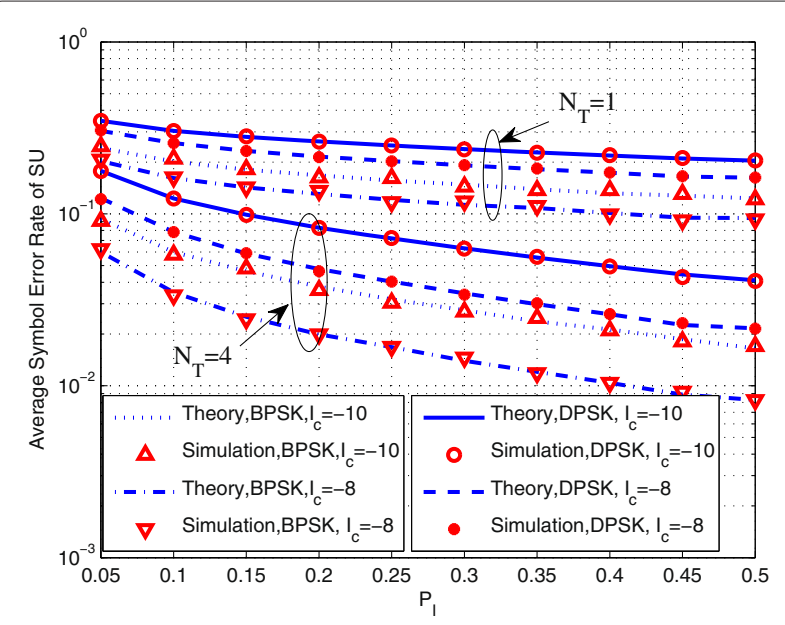

Fig. 9 The average symbol error rate vs. interference outage probability of SU: $P_{l}$, when $\rho=0.9$ and $P_{m}=0 \mathrm{dBW}$ 


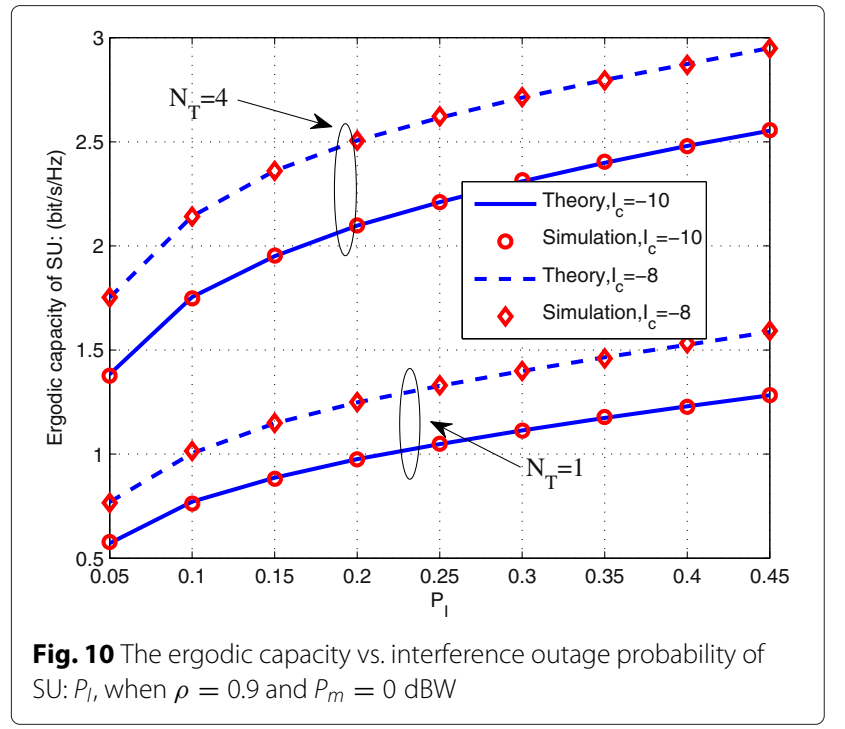

\section{Conclusions}

This article studies the performance of a TAS CR system in the presence of imperfect channel knowledge of the primary-secondary links under the peak interference constraint at PU and the maximum transmit power limit at SU. To protect the primary transmission, we apply a back-off control technique to restrict SU's power so that the interference probability of the primary transmission is satisfied. Utilizing the derived cumulative distribution function and probability density function of the SU's SNR, the closed form expressions of the secondary system performance are derived in terms of the average SER and capacity. Our theoretical analysis can serve as an efficient means to investigate the impacts of imperfect channel knowledge, the maximum transmit power limit, the interference power constraint, and the number of transmit antennas on the performance of secondary system. The theoretical analysis is validated by simulation results. Recent advances in CR networks have drawn the significance of security communications $[26,27]$. In our future works, we will research on performance analysis for security-based CR TAS networks.

\section{Competing interests}

The authors declare that they have no competing interests.

\section{Authors' contributions}

$\mathrm{PL}$ contributed to the conception and design of the study and writing of the manuscript. FS carried out the simulation and revised the manuscript. LC participated in the design of the study. GZ helped perform the analysis with constructive discussions and helped to draft the manuscript. All authors read and approved the final manuscript.

\section{Acknowledgements}

This work was supported in part by Shandong Province High School Science and Technology Fund Planning Project (J12LN02) and Research Fund for the Doctoral Program of Higher Education of China (20123702120016).

\section{Author details}

${ }^{1}$ College of Information Science and Engineering, Shandong Agricultural University, Daizong Road No.61, 271018 Tai'an, Shandong, China.
${ }^{2}$ Administration Center of Shandong Academy of Information and Communication Technology, Xinluo Street No. 1768 High-tech Zone, 250101 Jinan, China.

Received: 5 June 2015 Accepted: 6 June 2016

Published online: 16 June 2016

\section{References}

1. J Mitola, GQ Maguire, Cognitive radio: making software radios more personal. IEEE Pers. Commun. 6(4), 13-18 (1999)

2. Q Zhao, BM Sadler, A survey of dynamic spectrum access. IEEE Signal Process. Mag. 24(3), 79-89 (2007)

3. G Scutari, D Palomar, S Barbarossa, Cognitive MIMO radio. IEEE Signal Process. Mag. 25(6), 46-59 (2008)

4. Y Wang, J Coon, Difference antenna selection and power allocation for wireless cognitive systems. IEEE Trans. Commun. 59(12), 3494-3503 (2011)

5. MF Hanif, PJ Smith, DP Taylor, PA Martin, MIMO cognitive radios with antenna selection. IEEE Trans. Wireless Commun. 10(11), 3688-3699 (2011)

6. Z Chen, J Yuan, B Vucetic, Analysis of transmit antenna selection/maximal-ratio combining in Rayleigh fading channels. IEEE Trans. Veh. Technol. 54(4), 1312-1321 (2005)

7. L Yang, J Qin, Performance of Alamouti scheme with transmit antenna selection for M-ary signals. IEEE Trans. Wireless Commun. 5(12), 3365-3369 (2006)

8. H Wang, J Lee, S Kim, D Hong, Capacity enhancement of secondary links through spatial diversity in spectrum sharing. IEEE Trans. Wireless Commun. 9(2), 494-499 (2010)

9. V Blagojevic, P Ivanis, Ergodic capacity for TAS/MRC spectrum sharing cognitive radio. IEEE Commun. Lett. 16(3), 321-323 (2012)

10. FA Khan, KTourki, MS Alouini, KA Qaraqe, Performance analysis of a power limited spectrum sharing system with TAS/MRC. IEEE Trans. Sign. Process. 62(4), 954-967 (2014)

11. KTourki, FA Khan, KA Qaraqe, HC Yang, MS Alouini, Exact performance analysis of MIMO cognitive radio systems using transmit antenna selection. IEEE J. Sel. Areas Commun. 32(3), 425-438 (2014)

12. HA Suraweera, PJ Smith, M Shafi, Capacity limits and performance analysis of cognitive radio with imperfect channel knowledge. IEEE Trans. Veh. Technol. 59(4), 1811-1822 (2010)

13. VNQ Bao, TQ Duong, C Tellambura, On the performance of cognitive underlay multihop networks with imperfect channel state information. IEEE Trans. Commun. 61(12), 4864-4873 (2013)

14. X Jia, D Wang, X Dang, L Yang, H Zhu, Performance analysis of cooperative cognitive MIMO multiuser downlink transmission with perfect and imperfect CSI over Rayleigh fading channels. EURASIP J. Wireless Commun. Netw. 2015, 1-21 (2015)

15. S Tiiro, K Umebayashi, J Lehtomaki, Y Suzuki, Spectrum sharing in MIMO cognitive radio systems with imperfect channel state information. IEICE Trans. Commun. E97-B(4), 867-874 (2014)

16. D Li, Effect of channel estiamtion errors on arbitrary transmit antenna selection for cognitive MISO systems. IEEE Commun. Lett. 15(6), 656-658 (2011)

17. TQ Duong, PL Yeoh, VNQ Bao, M Elkashlan, N Yang, Cognitive relay networks with multiple primary transceivers under spectrum-sharing. IEEE Sign. Process. Lett. 19(11), 741-744 (2012)

18. J Lee, H Wang, JH Andrews, D Hong, Outage probability of cognitive relay networks with interference constraints. IEEE Trans. Wireless Commun. 10(2), 390-395 (2011)

19. TW Ban, W Choi, BC Jung, DK Sung, Multi-user diversity in a spectrum sharing system. IEEE Trans. Wireless Commun. 8(1), 102-106 (2009)

20. Q Wu, Y Huang, J Wang, Y Cheng, Effective capacity of cogntive radio systems with GSC diversity under imperfect channel knowledge. IEEE Commun. Lett. 16(11), 1792-1795 (2012)

21. H Kim, H Wang, S Lim, D Hong, On the impact of outdated channel information on the capacity of secondary user in spectrum sharing environments. IEEE Trans. Wireless Commun. 11(1), 284-293 (2012)

22. AH Nuttall, Some integrals involving the Q-function. (Naval Underwater Syst. Cent, New London, CT, Tech. Rep. 4297, 1971)

23. Y Chen, C Tellambura, Distribution functions of selection combiner output in equally correlated rayleigh, rician, and nakagami-m fading channels. IEEE Trans. Commun. 52(11), 1948-1956 (2004) 
24. JG Proakis, Digital Communications, 5th edition. (Mc Graw-Hill, New York, 2008)

25. IS Gradshteyn, IM Ryzhik, Table of Integrals, Series and Products, 7th edition. (Academic Press, New York, 2007)

26. Y Zou, J Zhu, L Yang, YC Liang, YD Yao, Securing physical-layer communications for cognitive radio networks. IEEE Commun. Maganize. 53(9), 48-54 (2015)

27. Y Zou, B Champagne, WP Zhu, L Hanzo, Relay-selection improves the security-reliability trade-off in cognitive radio systems. IEEE Trans. Commun. 63(1), 215-228 (2015)

\section{Submit your manuscript to a SpringerOpen ${ }^{\circ}$ journal and benefit from:}

- Convenient online submission

Rigorous peer review

- Immediate publication on acceptance

- Open access: articles freely available online

- High visibility within the field

- Retaining the copyright to your article

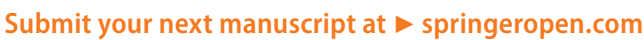

